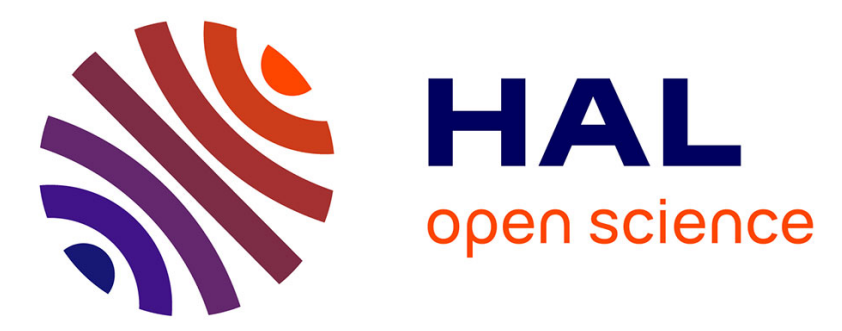

\title{
Seasonality and the evolutionary divergence of plant parasites
}

Frédéric Marie Hamelin, Magda Castel, Sylvain Poggi, Didier Andrivon, Ludovic Mailleret

\section{- To cite this version:}

Frédéric Marie Hamelin, Magda Castel, Sylvain Poggi, Didier Andrivon, Ludovic Mailleret. Seasonality and the evolutionary divergence of plant parasites. Ecology, 2011, 92 (12), pp.2159-2166. 10.1890/102442.1. hal-00729263

\section{HAL Id: hal-00729263}

\section{https://institut-agro-rennes-angers.hal.science/hal-00729263}

Submitted on 15 Nov 2012

HAL is a multi-disciplinary open access archive for the deposit and dissemination of scientific research documents, whether they are published or not. The documents may come from teaching and research institutions in France or abroad, or from public or private research centers.
L'archive ouverte pluridisciplinaire HAL, est destinée au dépôt et à la diffusion de documents scientifiques de niveau recherche, publiés ou non, émanant des établissements d'enseignement et de recherche français ou étrangers, des laboratoires publics ou privés. 


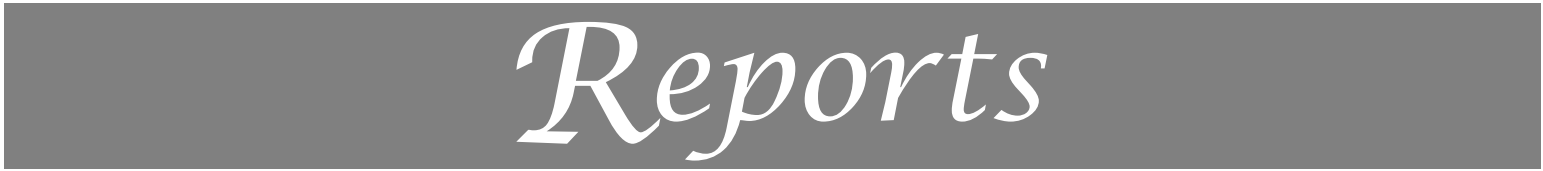

Ecology, 92(12), 2011, pp. 2159-2166

(c) 2011 by the Ecological Society of America

\title{
Seasonality and the evolutionary divergence of plant parasites
}

\author{
Frédéric M. Hamelin, ${ }^{1,5}$ Magda Castel, ${ }^{1}$ Sylvain Poggi, ${ }^{2}$ Didier Andrivon, ${ }^{2}$ and Ludovic Mailleret $^{3,4}$ \\ ${ }^{1}$ Agrocampus Ouest, UMR1099 BiO3P, 35042 Rennes, France \\ ${ }^{2}$ INRA, UMR1099 BiO3P, 35653 Le Rheu, France \\ ${ }^{3}$ INRA, UR880 URIH, 06903 Sophia Antipolis, France \\ ${ }^{4}$ INRIA, BIOCORE, 06902 Sophia Antipolis, France
}

\begin{abstract}
The coexistence of closely related plant parasites is widespread. Yet, understanding the ecological determinants of evolutionary divergence in plant parasites remains an issue. Niche differentiation through resource specialization has been widely researched, but it hardly explains the coexistence of parasites exploiting the same host plant. Time-partitioning has so far received less attention, although in temperate climates, parasites may specialize on either the early or the late season. Accordingly, we investigated whether seasonality can also promote phenotypic divergence. For plant parasites, seasonality generally engenders periodic host absence. To account for abrupt seasonal events, we made use of an epidemic model that combines continuous and discrete dynamics. Based on the assumption of a trade-off between in-season transmission and inter-season survival, we found through an "evolutionary invasion analysis" that evolutionary divergence of the parasite phenotype can occur. Since such a trade-off has been reported, this study provides further ecological bases for the coexistence of closely related plant parasites. Moreover, this study provides original insights into the coexistence of sibling plant pathogens which perform either a single or several infection cycles within a season (mono- and polycyclic diseases, or uni- and multivoltine life cycles).

Key words: adaptive dynamics; parasite; seasonality; semi-discrete model; sympatric speciation; voltinism.
\end{abstract}

\section{INTRODUCTION}

A fair fraction of present-day ecosystems are agricultural. Such ecosystems are characterized by the cyclical presence and absence of the main primary producer species. This special feature has ecological and evolutionary consequences for the community supported by this producer. In this paper, we have focused on crop parasites, often fungi, hereafter referred to as plant parasites.

The coexistence of closely related plant parasites (sibling species or genetically distinct subgroups within a species) is all pervasive (e.g., Fitt et al. 2006, Fournier and Giraud 2008, Daval et al. 2010, Montarry et al. 2008, Mougou Hamdane et al. 2010). This apparently challenges the competitive-exclusion principle, which states that "two species occupying the same ecological niche cannot coexist indefinitely" (Gause 1934). Under-

Manuscript received 23 December 2010; revised 6 May 2011; accepted 1 July 2011. Corresponding Editor: A. M. de Roos.

${ }^{5}$ E-mail: fhamelin@agrocampus-ouest.fr standing the ecological determinants of evolutionary divergence in plant parasites is an issue that pertains to both evolutionary ecology and agricultural sciences (Fitt et al. 2006, Giraud et al. 2010). Ecological differences that lead to niche partitioning can occur in three basic ways: resource specialization, time partitioning, and space partitioning (Amarasekare 2003). Spatial partitioning can occur at small scales (microhabitat differentiation) or at large scales (geographical differentiation). In this respect, Fitt et al. (2006) referred to separation in space as microhabitat differentiation (e.g., stem base or upper stem lesions), which is often associated with separation in time or in resource use.

Niche differentiation through resource specialization has been thoroughly understood, thanks to adaptive dynamics theory. (For an introduction to concepts, ideas, and methods developed by the authors of Metz et al. [1992, 1996], Dieckmann and Law [1996], Geritz et al. [1998], and others, see Diekmann [2004].) Schreiber and Tobiason (2003) showed that evolutionary divergence (henceforth referred to as evolutionary branching) can occur within the context of resource use. Regarding 
plant parasites, Gudelj et al. $(2004 a, b)$ showed that evolutionary branching can occur, provided there is a (convex) trade-off between parasite transmission capacities on distinct host types. Evolutionary dynamics then lead to complete host specialization. However, closely related plant parasites can have overlapping host ranges (Fitt et al. 2006). In this respect, Gandon (2004) showed that low values of inter-host-type transmissions can promote evolutionary branching, provided that the optimal within-host exploitation strategies are distinct. Regarding the coexistence of parasites sharing a single host, several authors showed that multiple infections, which endogenously generate host heterogeneity, may also promote evolutionary branching (Alizon and van Baalen 2008, Boldin and Diekmann 2008).

To date, time-partitioning has received less attention, although in temperate climates, parasites may specialize either on the early or on the late season. Seasonality, possibly combined with agricultural practices such as harvesting and planting, generally engenders periodic host absence. Indeed, many parasites are subjected to host absence during winter or summer seasons. Thus, we investigated whether periodic host absence can promote evolutionary branching.

Although Shaw (1994) stressed that periodic host absence can induce major qualitative changes in plant parasite population dynamics such as chaos, it is only recently that this feature has been incorporated into evolutionary analyses. Interestingly, Koelle et al. (2005) investigated parasite adaptation to seasonal forcing, but restricted their analysis to monomorphic evolutionary endpoints. Tachikawa (2008) showed that fluctuations in the resource supply can induce evolutionary branching in a microbial ecosystem, but niche differentiation through time partitioning was not observed.

To investigate the possibility of evolutionary branching due to periodic host absence, van den Berg et al. $(2010,2011)$ introduced a framework that makes use of epidemic models combining continuous and discrete dynamics to capture abrupt seasonal events (Geritz and Kisdi 2004, Mailleret and Lemesle 2009, Akhmetzhanov et al. 2011). Through evidence gathered from several host-parasite models (Carson 1998, Abang 2006), these authors considered a trade-off between in-season transmission and inter-season survival. In a set of generic models, van den Berg et al. (2010, 2011) demonstrated that an optimization principle holds; i.e., evolution maximizes some quantity that one may name fitness (Metz et al. 1992, 2008, Diekmann 2004, Gyllenberg and Service 2011). As a point of interest, this implies competitive exclusion and precludes the possibility of evolutionary branching.

In addition, van den Berg et al. (2010) considered a particular feature of plant disease epidemics: the cooccurrence of primary and secondary infections. Primary infections are generated by the primary inoculum, i.e., the form under which the parasite survives host absence. Secondary infections are those subsequently generated by infected hosts. Based on numerical simulations, these authors concluded that "periodicity in host availability does not account for evolutionary branching, as observed in many plant pathogens." In this paper, we have revisited the issue of plant parasite evolution in temperate environments and shown that periodic host absence indeed promotes evolutionary branching, provided there is a trade-off between in-season transmission and inter-season survival.

\section{ECo-eVolutionary Model}

This section introduces an ecological model very similar to Madden and van den Bosch (2002) and sets the scene for an evolutionary invasion analysis sensu adaptive dynamics theory; this entails the derivation of an invasion criterion for a rare mutant subpopulation, challenging a phenotypically distinct resident population (Diekmann 2004).

\section{Ecological model}

Let $(P, S, I)$ denote the primary inoculum, susceptible host, and infected host densities, respectively. $T$ denotes the length of one cycle (e.g., one year), $\tau<T$ the length of the period during which the host is present, and $n$ a cycle index. Moreover, let $\Theta$ and $\beta$ be the primary and secondary-infection rate constants, respectively. In addition, let $\beta_{\max }$ be the biologically feasible maximum secondary-infection rate constant, i.e., $0 \leq \beta \leq \beta_{\max }$. Last, we assume that the primary inoculum has a specific inter-season (e.g., winter) mortality rate $\mu$. Among plant parasites, there is biological evidence of a trade-off between in-season transmission and inter-season survival (Carson 1998, Abang et al. 2006) that, similarly to van den Berg et al. $(2010,2011)$, we model as $\mu=f(\beta)$, with $f(\cdot)$ an increasing function. Since the adaptive dynamics theory is concerned with whether a small mutant subpopulation can invade the resident population, two parasite compartments have to be distinguished. Let the subscript $i=1$, 2 denote the resident and the mutant population densities $\left(P_{i}\right.$ and $\left.I_{i}\right)$ and traits $\left(\beta_{i}\right.$ and $\left.\mu_{i}\right)$, respectively.

Full model.-The plant epidemic system under study is characterized by a temporal cycle composed of two time periods during which host plants are present or absent, respectively; these time periods are separated by discrete events such as crop harvest or planting. Fig. 1 displays a diagram representing the epidemic processes that are further detailed in what follows.

1. During host presence.-We consider a basic SIR model (Smith 2008; governing secondary-infection dynamics) with additional primary-infection dynamics, which is standard in plant epidemiology (Madden and van den Bosch 2002, Madden et al. 2007, van den Berg et al. 2010). Let $\alpha$ denote the per capita rate at which infected hosts are removed from the epidemiological dynamics and $\Lambda$ be a primary inoculum loss rate. Introducing the notations $\dot{S}=d S / d t$ and $t^{-}, t^{+}$to denote the instants right before and right after $t$, the model reads as follows for $i=1,2$, for all $t$ between $n T$ and $n T+\tau$ : 


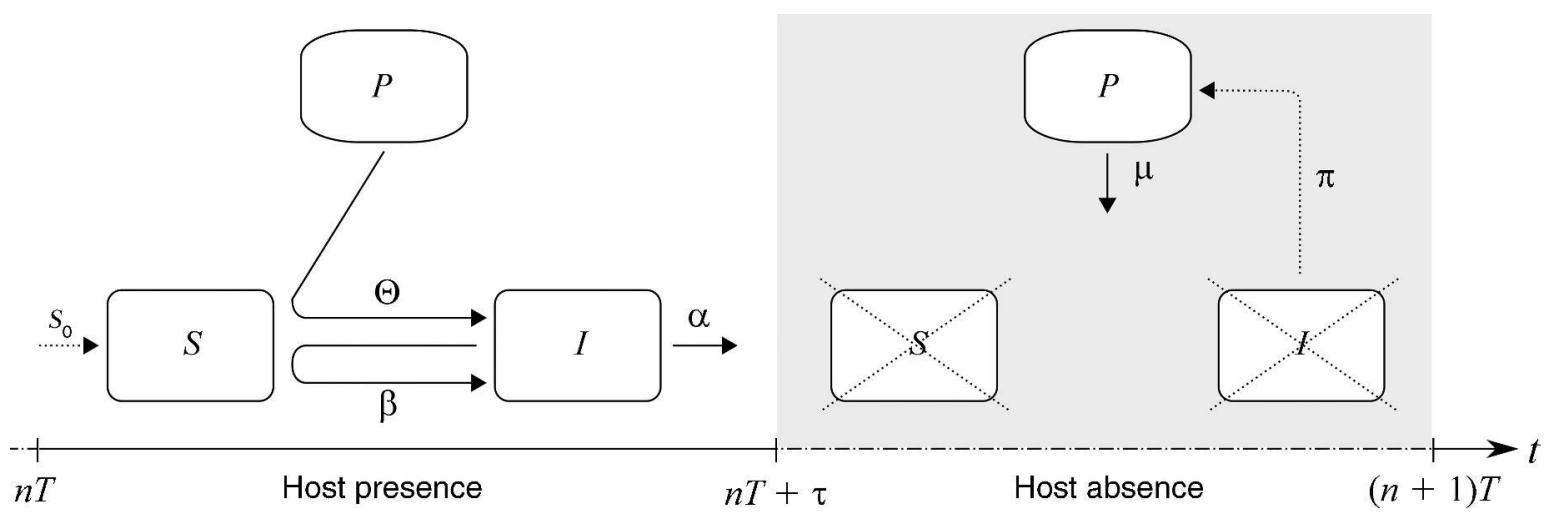

FIG. 1. Schematic representation of the course of an epidemic over one cycle of length $T$. Continuous phenomena (primary and secondary infections, primary inoculum mortality) are represented by plain lines, whereas discrete phenomena (planting, harvest, conversion of infected individuals into survival forms) are represented by dotted lines. $P, S$, and $I$ denote the primary inoculum, susceptible host, and infected host densities, respectively, $T$ denotes the length of one cycle (e.g., one year), $\tau<T$ is the length of the period during which the host is present, and $n$ is a cycle index. $S_{0}$ is the density of susceptible hosts at the beginning of each new season. The variables $\Theta$ and $\beta$ are the primary- and secondary-infection rate constants, respectively. Inter-season (e.g., winter) mortality rate is $\mu ; \alpha$ denotes the per capita rate at which infected hosts are removed from the epidemiological dynamics; time is $t$; infected hosts convert into primary inoculum with a conversion factor $\pi$. Crossed-out boxes represent host removal.

$$
\begin{array}{cccc}
\dot{P}_{i} & = & -\Lambda P_{i} & \\
\dot{S} & = & -\sum_{i} \Theta P_{i} S & -\sum_{i} \beta_{i} S I_{i} \\
\dot{I}_{i} & = & +\Theta P_{i} S & +\beta_{i} S I_{i}-\alpha I_{i} . \\
& & \text { primary infections } & \text { secondary infections }
\end{array}
$$

The first-column terms of the right hand side of this equation model primary infections; they indicate that only a fraction of the released primary inoculum actually encounters healthy hosts and initiates primary infections, while the remaining part is lost. The second column corresponds to the basic SIR model, which describes secondary infections.

2. Transition from host presence to host absence.-At time $t=(n T+\tau)$, hosts are removed (e.g., harvested) and infected hosts convert into primary inoculum with a conversion factor $\pi$. For $i=1,2$,

$$
\begin{aligned}
P_{i}\left(n T+\tau^{+}\right) & =P_{i}\left(n T+\tau^{-}\right)+\pi I_{i}\left(n T+\tau^{-}\right) \\
S\left(n T+\tau^{+}\right) & =0 \\
I_{i}\left(n T+\tau^{+}\right) & =0
\end{aligned}
$$

In other words, after harvest, the parasite that is still present on crop debris switches to a survival form (Madden and van den Bosch 2002, Agrios 2005, van den Berg et al. 2010, 2011).

3. During host absence.-The parasite has a specific between-season mortality rate $\mu_{i}$ for $i=1,2$, for all $t$ between $(n T+\tau)$ and $(n+1) T$ :

$$
\dot{P}_{i}=-\mu_{i} P_{i} \quad \dot{S}=0 \quad \dot{I}_{i}=0 .
$$

4. Transition from host absence to host presence.-At the beginning of each new season (time $t=[n+1] T$ ), a density $S_{0}$ of susceptible hosts is made available to the parasite (e.g., planted):

$$
\begin{aligned}
P_{i}\left([n+1] T^{+}\right) & =P_{i}\left([n+1] T^{-}\right) \\
S\left([n+1] T^{+}\right) & =S_{0} \\
I_{i}\left([n+1] T^{+}\right) & =0 .
\end{aligned}
$$

Reduction to a compact model.-As suggested by Madden and van den Bosch (2002), it may be assumed that primary-infection dynamics occur on a faster time scale than secondary-infection dynamics. On the basis of this assumption, the theory of slow-fast dynamical systems can be used to reduce the dimension of the model. This will make the model more tractable for the evolutionary invasion analysis to be performed. Let us summarize what we show in greater detail in Mailleret et al. (2011). Let $0<\varepsilon \ll 1$ be the scaling factor between the slow and the fast time scales (times $t$ and $z$ $=t / \varepsilon$, respectively), $\theta=\varepsilon \Theta$, and $\lambda=\varepsilon \Lambda$. Considering the fast time scale (time $z$ ) by introducing the notation $S^{\prime}=$ $d S / d z$ and dropping first-order terms in $\varepsilon$, one gets, for $i=1,2$,

$$
\begin{aligned}
& P_{i}^{\prime}=-\lambda P_{i} \\
& S^{\prime}=-\sum_{i} \theta S P_{i} \\
& I_{i}^{\prime}=+\theta S P_{i}
\end{aligned}
$$

so that for $i=1,2,\left[P_{i}, S, I_{i}\right]$ very rapidly converge to

$$
P_{i}=0 \quad S=S_{0} \exp \left(-\sum_{i} \frac{\theta P_{i}\left(n T^{+}\right)}{\lambda}\right)
$$

$$
I_{i}=S_{0}\left[1-\exp \left(-\sum_{i} \frac{\theta P_{i}\left(n T^{+}\right)}{\lambda}\right)\right] \frac{\frac{\theta P_{i}\left(n T^{+}\right)}{\lambda}}{\sum_{i} \frac{\theta P_{i}\left(n T^{+}\right)}{\lambda}}
$$


These values will be used as initial conditions of the secondary-infection dynamics which are governed by the remaining part of Eq. 1, as compared to Eq. 5. Before doing this, it is to be noted that one can solve Eqs. 2, 3, and 4 , leading to

$$
\begin{aligned}
P_{i}\left([n+1] T^{+}\right) & =\pi e^{-\mu_{i}(T-\tau)} I_{i}\left(n T+\tau^{-}\right) \\
S\left([n+1] T^{+}\right) & =S_{0} \\
I_{i}\left([n+1] T^{+}\right) & =0 .
\end{aligned}
$$

Eq. 6 indeed shows that the primary inoculum is fully depleted following the fast primary-infection phase; hence, $P_{i}\left(n T+\tau^{-}\right)=0$.

The model thus reduces to a standard SIR epidemic model: for all $t$ between $n T$ and $n T+\tau$,

$$
\begin{aligned}
& \dot{S}=-\sum_{i} \beta_{i} I_{i} S \\
& \dot{I}_{i}=+\beta_{i} S I_{i}-\alpha I_{i}
\end{aligned}
$$

with discrete cycle-to-cycle dynamics:

$S([n+1] T)=S_{0} e^{-\sum_{i} F_{i}(n T+\tau)}$

$I_{i}([n+1] T)=S_{0}\left(1-e^{-\sum_{i} F_{i}(n T+\tau)}\right) \frac{F_{i}(n T+\tau)}{\sum_{i} F_{i}(n T+\tau)}$

where

$$
F_{i}(n T+\tau)=\frac{\theta \pi e^{-\mu_{i}(T-\tau)}}{\lambda} I_{i}(n T+\tau)
$$

will be referred to as the primary-infection force.

The model in Eqs. 7-9 provides a very good approximation of the model in Eqs. 1-4 as long as $\varepsilon$ is small (Mailleret et al. 2011). From now on, we make use of this reduced model as a proxy to investigate the evolutionary implications of the full model.

\section{Evolutionary invasion analysis}

Although the model in Eqs. 7-9 can show chaotic dynamics, it turns out that in a large part of the parameter space, asymptotic solutions are $T$ periodic (Mailleret et al. 2011). Thus, let us assume that a resident trait $\beta_{1}$ generates a $T$ periodic mutant-free solution $\left[S^{\circ}\left(t, \beta_{1}\right), I_{1}^{\circ}\left(t, \beta_{1}\right), 0\right]$, which, for brevity, we also call endemic equilibrium. Let

$$
\bar{S}^{\circ}\left(\beta_{1}\right)=\frac{1}{\tau} \int_{0}^{\tau} S^{\circ}\left(t, \beta_{1}\right) \mathrm{d} t
$$

denote the mean healthy host density, at equilibrium. In addition, let

$$
F_{1}^{\circ}\left(\beta_{1}\right)=\frac{\theta \pi e^{-\mu_{1}(T-\tau)}}{\lambda} I_{1}^{\circ}\left(\tau, \beta_{1}\right) .
$$

We are interested in finding out whether a mutant can invade the resident population at equilibrium. Since we focus on the fate of a very small mutant subpopulation $\left(I_{2} \ll I_{1}\right)$, we have $\sum_{i} F_{i} \cong F_{1}^{\circ}\left(\beta_{1}\right)$. Eq. 8 therefore reads $I_{2}([n+1] T) \cong S_{0}\left(1-e^{-F_{1}^{\circ}}\right) F_{2} / F_{1}^{\circ}$. Assuming that the mutant phenotype is rare, or that it has little effect on the $S$ dynamics as shaped by the resident, reads $I_{2}([n T+$ $\tau] \cong I_{2}(n T) \exp \left(\left[\beta_{2} \bar{S}^{\circ}\left(\beta_{1}\right)-\alpha\right] \tau\right)$. Using the latter equation and Eq. 9, one finds that the mutant can invade provided that

$$
\begin{aligned}
\frac{I_{2}([n+1] T)}{I_{2}(n T)} \cong & S_{0} \frac{1-e^{-F_{1}^{\circ}\left(\beta_{1}\right)}}{F_{1}^{\circ}\left(\beta_{1}\right)} \frac{\theta \pi e^{-\mu_{2}(T-\tau)}}{\lambda} \\
& \quad \times \exp \left(\left[\beta_{2} \bar{S}^{\circ}\left(\beta_{1}\right)-\alpha\right] \tau\right)>1
\end{aligned}
$$

whereas if it is less than one, the mutant dies out. It is worthy to note that the above invasion criterion is bidimensional in the environment. In other words, two variables determined by the resident, the mean healthy host density $\bar{S}^{\circ}$ and the resident's primary-infection force $F_{1}^{\circ}$, determine the mutant's reproductive ratio. This precludes an optimization principle (see Introduction) and thus leaves room for evolutionary branching to occur (Metz et al. 2008, Gyllenberg and Service 2011). Note that in the limit case where the mutant does not differ from the resident, i.e., substituting $\mu_{2}$ and $\beta_{2}$ by $\mu_{1}$ and $\beta_{1}$ in Eq. 10, the inequality transforms into an equality (the mutant's reproductive ratio is equal to that of the resident, which is 1 since the resident population is at equilibrium). Using the latter equality and Eq. 10, the invasion criterion reads

$$
\frac{I_{2}([n+1] T)}{I_{2}(n T)} \cong \frac{\exp \left[\beta_{2} \tilde{S}^{\circ}\left(\beta_{1}\right) \tau-\mu_{2}(T-\tau)\right]}{\exp \left[\beta_{1} \tilde{S}^{\circ}\left(\beta_{1}\right) \tau-\mu_{1}(T-\tau)\right]}>1 .
$$

We have no explicit expression of $\bar{S}^{\circ}$ in this model (even in the $\alpha=0$ case). One can nevertheless perform numerical computations to determine the regions of the $\left(\beta_{1}, \beta_{2}\right)$ plane where the mutant invades, or does not invade. This is termed a pairwise invasibility plot (PIP) in the adaptive dynamics framework (Diekmann 2004).

\section{RESULTS}

Investigating whether evolutionary branching can occur requires further study on the invasion criterion Eq. 11 or the fitness of a rare mutant in the environment as shaped by the resident; this frequency dependent fitness concept is called invasion fitness in the adaptive dynamics terminology. Using the fact that for $i=1,2, \mu_{i}$ $=f\left(\beta_{i}\right)$, Eq. 11 yields the following invasion fitness function, which is positive if the mutant can invade:

$$
s\left(\beta_{1}, \beta_{2}\right)=\left(\beta_{2}-\beta_{1}\right) \bar{S}^{\circ}\left(\beta_{1}\right) \tau-\left[f\left(\beta_{2}\right)-f\left(\beta_{1}\right)\right](T-\tau) .
$$

Alternatively, if $s\left(\beta_{1}, \beta_{2}\right)<0$, the invading mutant population will die out. An evolutionary singular trait $\beta^{\star}$ is such that the local invasion fitness gradient is zero, i.e., $\mathrm{D}_{2} s\left(\beta^{\star}, \beta^{\star}\right)=0$, where $\mathrm{D}_{2} s$ means the partial derivative with respect to the second argument of $s$. 
FIG. 2. (A, B) Pairwise invasibility plots. Areas where the mutant can invade are in gray. Parameter values (which we compare to the corresponding take-all disease of wheat parameter values, from Bailey and Gilligan [1999] and van den Berg et al. [2011]) were: $S_{0}=1$ arbitrary host plant unit, $\tau=200 \mathrm{~d}$ (184 d in van den Berg et al. [2011]), $T=365 \mathrm{~d}, \alpha=0.005 \mathrm{~d}^{-1}$ (within $0-$ 0.05 , according to Bailey and Gilligan [1999] and van den Berg et al. [2011]), $\pi=0.5$ (half of the infectious roots become primary inoculum units, right after harvest), $\theta=0.5$ per day per primary inoculum unit $(0.6$, as estimated from Bailey and Gilligan [1999]), $\lambda=0.05 \mathrm{~d}^{-1}$ (within 0.04-0.06, according to Bailey and Gilligan [1999]), $\beta_{\max }=$ 0.1 (same order of magnitude as $\theta$, hosts being roots as well), and $\mu=f(\beta)=c \beta^{a}$ with coefficient $c=0.1$ and shape parameter $a$ either $a=1.2$ (convex trade-off) or $a=0.8$ (concave trade-off). (An intermediate $\mu$ value thus is $0.1 \times 0.05^{1}=$ 0.005, comparable to van den Berg et al.'s [2011] $\mu^{\prime}=0.007$.) $(\mathrm{C}, \mathrm{D})$ The associated evolutionary dynamics. Numerical computations were realized using an algorithm which is described in the body of the paper. (E, F) Ecological dynamics at evolutionary endpoints, from the full model $(\varepsilon$ $=0.05)$. For all panels, $\beta_{\max }$ is the biologically feasible maximum secondary-infection rate constant, $\varepsilon$ is the scaling factor, $\theta=\varepsilon \Theta$, and $\lambda=\varepsilon \Lambda$ (where $\Lambda$ is a primary inoculum loss rate). An evolutionarily singular trait $\beta^{\star}$ is such that the local invasion fitness gradient is zero.
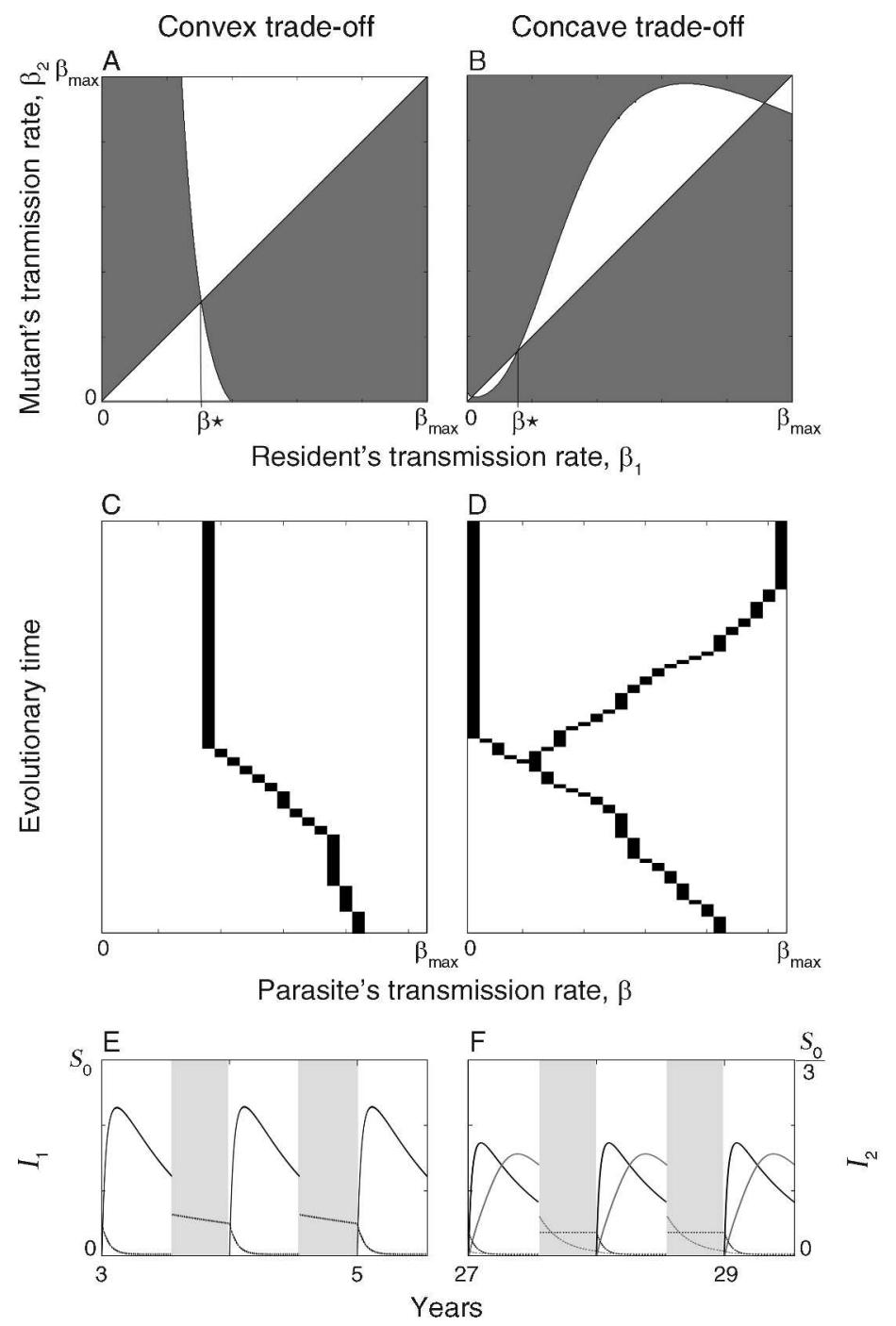

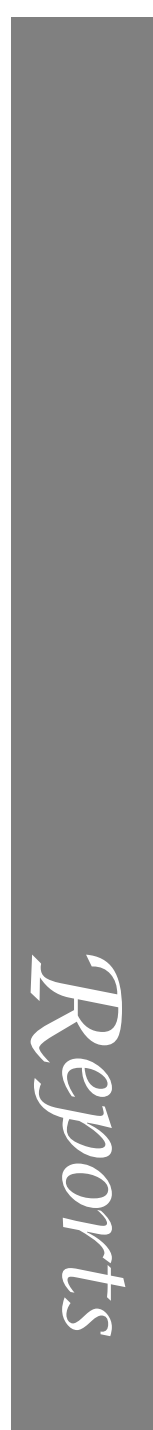

Several evolutionary outcomes are possible, depending on the sign of the invasion fitness function around a singular point. If the singular point repels, it is called an evolutionary repeller. On the other hand, if the singular point is both attractive and uninvasible, it is called a continuously stable strategy. As a point of interest, the singular point can be attractive yet invasible, in which case it is called an evolutionary branching point.

One necessary condition for evolutionary branching to occur reads $\mathrm{D}_{22} s\left(\beta^{\star}, \beta^{\star}\right)>0$, which means that $s\left(\beta^{\star}, \beta^{\star}\right)$ is at a minimum (as opposed to at a maximum) in the adaptive landscape and that the trait $\beta^{\star}$ is thus invasible (as opposed to uninvasible). We have $\mathrm{D}_{22} s(\beta, \beta)=$ $-f^{\prime \prime}(\beta)(T-\tau)$, which shows that the concavity of the trade-off function completely determines whether a singular point is uninvasible or not (a branching point if it is also evolutionarily attracting). Fig. 2 illustrates this. (We checked that mutant-free dynamics are $T$ periodic for all shown $\beta$ values before drawing the PIPs.) Starting from the upper left panel, one sees that a convex trade-off leads to a monomorphic endpoint of evolution. As soon as the trade-off is concave, evolution converges toward a point where both greater and lower trait values can invade (Fig. 2B). This is a branching point. It is surrounded by two evolutionary repellers which demarcate its basin of attraction.

When the population becomes dimorphic, PIP formalism is no longer appropriate. One can nevertheless perform further evolutionary computations. Those in Fig. 2C, D were realized from the model in Eqs. 7-9 using the following algorithm. The evolving phenotype $\beta$ ranges from 0 to $\beta_{\max }$. This interval is divided into a finite number of subintervals (here 25). Starting from a monomorphic population having a certain $\beta$ value, evolutionary dynamics are governed by the following iteration rule. Once at ecological equilibrium, a small mutation occurs, having an equal probability of being on the left (smaller $\beta$ ) or on the right (larger $\beta$ ) of the subinterval under consideration. There are three possible outcomes: (1) non-invasion, when the mutant is 
excluded by the resident, (2) substitution, when the mutant excludes the resident, and (3) coexistence, when the mutant and the resident phenotypes do not exclude each other. The latter outcome can lead to evolutionary branching, by which the population may reach a dimorphic steady state.

For a convex trade-off, the singular trait $\beta^{\star}$ is shown to be a monomorphic endpoint of the evolutionary dynamics (Fig. 2C). For a concave trade-off, evolutionary dynamics first converge to $\beta^{\star}$ (the branching point), after which the parasite population splits into two groups, whose phenotypes diverge (Fig. 2D). Once the phenotypic boundaries 0 and $\beta_{\max }$ are reached, evolutionary dynamics remain steady. Thus, the evolutionary endpoint is dimorphic only. (This actually results from the fact that the environment is two-dimensional in Eq. 10, so there exists no trimorphic coexistence region.) Fig. $2 \mathrm{E}$ and $\mathrm{F}$ show the corresponding disease dynamics from the model in Eqs. 1-4.

\section{Discussion}

Fitt et al. (2006) proposed several mechanisms enabling coexistence of closely related plant parasites to occur: separation in time (one parasite occurs earlier in the growing season than the other), separation in resource use (e.g., the ability to colonize living or dead plant tissues), and separation in space (e.g., stem base or upper stem lesions), which actually corresponds to microhabitat differentiation, and is often associated to time or resource partitioning. Several authors showed that resource specialization can promote evolutionary divergence and coexistence of parasites (Gandon 2004, Gudelj et al. 2004a, b, Alizon and van Baalen 2008, Boldin and Diekmann 2008). Separation in time has received less attention so far, although in plant parasites, there is no lack of empirical evidence (e.g., Montarry et al. 2008). In a recent study, van den Berg et al. $(2010,2011)$ introduced a framework to investigate whether periodic host absence can promote evolutionary branching, and reached the conclusion that it cannot.

Our results challenge this view. Using standard model reduction techniques in mathematical ecology, we were able to explore a seasonal plant epidemic model which explicitly incorporates primary inoculum and infection dynamics. This allowed us to show that negative density dependence naturally arises in the season-to-season dynamics, while it is absent from (van den Berg et al. 2011). Since the way density dependence limits population growth generally matters in evolutionary ecology (Mylius and Diekmann 1995), this makes it a relevant difference. We have also shown that a necessary condition for evolutionary branching to occur is that the trade-off between in-season transmission and inter-season mortality has a concave shape. Further numerical computations indeed showed that evolutionary branching is possible within this framework.
We have also shown that the dimorphic evolutionary endpoint corresponds to the coexistence of parasites exploiting a single host and either minimizing or maximizing the secondary-infection rate constant. In accordance with the considered evolutionary trade-off, the former parasite thus survives winter better, and focuses on the primary-infection transmission route. Conversely, the latter parasite almost gives up winter survival to focus on the secondary-infection transmission route. Thus, evolutionary branching leads to the interplay of primary and mostly secondary infectors (Fig. 2E, F). Such epidemiological dynamics are typical of those observed on the grapevine powdery mildew, Erysiphe necator, for which two genetically distinct parasite strains coexist. Montarry et al. (2008) showed that niche partitioning, enabling the coexistence of two genetically differentiated groups of E. necator isolates (A and B) on the same host (Vitis vinifera), is caused by separation in time, as opposed to separation in space. The temporal dynamics showed that group A isolates were active only at the beginning of the growing season and disappeared during the course of the epidemic, whereas group B isolates were responsible for late infections.

Our model has also shown how mono- and polycyclic parasites (single and multiple infection cycles within a season, respectively [Agrios 2005, Madden et al. 2007]) may have diverged during the course of evolution. Monocyclic parasites may have traded secondaryinfection ability for primary-infection efficiency, through inter-season survival. Fitt et al. (2006) illustrated separation in time with the sibling species Mycosphaerella brassicicola (ring spot) and M. capsellae (white leaf spot). These species cause coexisting leaf spots on oilseed rape leaves (Inman et al. 1991, Gudelj et al. 2004a). " M. capsellae [...] produces infective conidia and white leaf spot is a polycyclic disease, whereas $M$. brassicicola does not produce conidia and ring spot is a monocyclic disease." A way to test the theory would be to investigate whether there is a trade-off between survival and infection efficiency in these sibling species.

From a broader ecological perspective, our results also shed light on the evolution of voltinism, or the number of generations an organism realizes within a year (uni- and multivoltine refer to single and several generations within a year). Alvarez et al. (2006) studied ecological niche differentiation within two sibling species of bean beetles, Acanthoscelides obtectus and $A$. obvelatus. According to the authors, the two sibling species coexist in sympatry, feed on the same host, and "the functional trait that best differentiates the two species is their difference in voltinism. Whereas $A$. obvelatus is [...] univoltine, A. obtectus is multivoltine." Alvarez et al. (2006) also report that "the higher frequency of A. obvelatus at the beginning of the season compared with its frequency 4 months later indicates a lower survival of $A$. obtectus during the rest of the year, 
as inter-annual frequencies are stable." Obligatory reproductive diapause in $A$. obvelatus and the impossibility for $A$. obtectus to enter reproductive diapause may be the two extremes of a trade-off between inter-season survival and in-season reproduction. Taking into consideration our study, it may be that the two sibling species have evolved in response to sympatric competition, rather than in allopatry, i.e., at different altitudes, as Alvarez et al. (2006) suggested.

\section{ACKNOWLEDGMENTS}

This research was supported by Agropolis Fondation and RNSC (covenant support number 0902-013), and INRA (contract number 394576). M. Castel is supported by a Ph.D. fellowship from the INRA "Plant Health and the Environment" Division and the Council of Région Bretagne. This work is part of an INRA-BBSRC funded project entitled "Epidemiological and evolutionary models for invasion and persistence of plant diseases." S. Poggi acknowledges the ANR for funding the SYSBIOTEL project referenced ANR-08_STRA-14. We thank F. van den Berg and F. van den Bosch for their comments on a previous version of the manuscript, D. J. Bailey, J. Montarry, M. Plantegenest, and A. Sarniguet for stimulating discussions, and two anonymous reviewers for insightful comments. Thanks to I. Mascio for her help in the English editing.

\section{Literature Cited}

Abang, M. M., M. Baum, S. Ceccarelli, S. Grando, C. C. Linde, A. Yahyaoui, J. Zhan, and B. A. McDonald. 2006. Differential selection on Rhynchosporium secalis during parasitic and saprophytic phases in the barley scald disease cycle. Phytopathology 96:1214-1222.

Agrios, G. N. Plant pathology. 2005. Elsevier Academic Press, Amsterdam, The Netherlands.

Akhmetzhanov, A. R., F. Grognard, and L. Mailleret. 2011. Optimal life history strategies in seasonal consumer-resource dynamics. Evolution. [doi: 10.1111/j.1558-5646.2011.01381.x]

Alizon, S., and M. van Baalen. 2008. Multiple infections, immune dynamics and the evolution of virulence. American Naturalist 172:E150-168.

Alvarez, N., L. Mercier, M. Hossaert-McKey, J. ContrerasGarduño, G. Kunstler, A. Aebi, and B. Benrey. 2006. Ecological distribution and niche segregation of two sibling species of bean beetles, Acanthoscelides obtectus Say and A. obvelatus Bridwell. Ecological Entomology 31:582-590.

Amarasekare, P. 2003. Competitive coexistence in spatially structured environments: a synthesis. Ecology Letters 6:1109-1122.

Bailey, D. J., and C. A. Gilligan. 1999. Dynamics of primary and secondary infection in take-all epidemics. Phytopathology 89:84-91.

Boldin, B., and O. Diekmann. 2008. Superinfections can induce evolutionarily stable coexistence of pathogens. Journal of Mathematical Biology 56:635-672.

Carson, M. L. 1998. Aggressiveness and perennation of isolates of Cochliobolus heterostrophus from North Carolina. Plant Disease 82:1043-1047.

Daval, S., L. Lebreton, K. Gazengel, A.-Y. Guillerm-Erckelboudt, and A. Sarniguet. 2010. Genetic evidence for differentiation of Gaeumannomyces graminis var. tritici into two major groups. Plant Pathology 59:165-178.

Dieckmann, U., and R. Law. 1996. The dynamical theory of coevolution. Journal of Mathematical Biology 34:579-612.

Diekmann, O. 2004. A beginners guide to adaptive dynamics. Pages 47-86 in R. Rudnicki, editor. Mathematical modelling of population dynamics. Banach Center Publications, Vol- ume 63. Institute of Mathematics, Polish Academy of Sciences, Warszawa, Poland.

Fitt, B. D. L., Y.-J. Huang, F. van den Bosch, and J. S. West. 2006. Coexistence of related pathogen species on arable crops in space and time. Annual Review of Phytopathology 44:163-182.

Fournier, E., and T. Giraud. 2008. Sympatric genetic differentiation of populations of a generalist pathogenic fungus, Botrytis cinerea, on two different host plants, grapevine and bramble. Journal of Evolutionary Biology 21:122-132.

Gandon, S. 2004. Evolution of multihost parasites. Evolution 58:455-469.

Gause, G. F. 1934. The struggle for existence. Williams and Wilkins Publishers, Baltimore, Maryland, USA.

Geritz, S. A. H., and E. Kisdi. 2004. On the mechanistic underpinning of discrete time population models with complex dynamics. Journal of Theoretical Biology 228:261269.

Geritz, S. A. H., E. Kisdi, G. Meszéna, and J. A. J. Metz. 1998. Evolutionarily singular strategies and the adaptive growth and branching of the evolutionary tree. Evolutionary Ecology 12:35-57.

Giraud, T., P. Gladieux, and S. Gavrilets. 2010. Linking emergence of fungal plant diseases and ecological speciation. Trends in Ecology and Evolution 25:387-395.

Gudelj, I., F. van den Bosch, and B. D. L. Fitt. $2004 a$. Evolution of sibling fungal plant pathogens in relation to host specialisation. Phytopathology 94:789-795.

Gudelj, I., F. van den Bosch, and C. A. Gilligan. $2004 b$. Transmission rates and adaptive evolution of pathogens in sympatric heterogeneous plant populations. Proceedings of the Royal Society B 272:2187-2194.

Gyllenberg, M., and R. Service. 2011. Necessary and sufficient conditions for the existence of an optimisation principle in evolution. Journal of Mathematical Biology 62:359-369.

Inman, A. J., A. Sivanesan, B. D. L. Fitt, and R. L. Evans. 1991. The biology of Mycosphaerella capsellae sp. nov., the teleomorph of Pseudocercosporella capsellae, cause of white leaf spot of oilseed rape. Mycological Research 95:13341342.

Koelle, K., M. Pascual, and M. Yunus. 2005. Pathogen adaptation to seasonal forcing and climate change. Proceedings of the Royal Society B 272:971-977.

Madden, L. V., G. Hughes, and F. van den Bosch. 2007. The study of plant disease epidemics. APS Press, St. Paul, Minnesota, USA

Madden, L. V., and F. van den Bosch. 2002. A populationdynamics approach to assess the threat of plant pathogens as biological weapons against annual crops. BioScience 52:6574.

Mailleret, L., M. Castel, J. Montarry, and F. M. Hamelin. 2011. From elaborate to compact seasonal plant epidemic models and back; is competitive exclusion in the details? Theoretical Ecology. [doi: 10.1007/s12080-011-0126-0]

Mailleret, L., and V. Lemesle. 2009. A note on semi-discrete modelling in the life sciences. Philosophical Transactions of the Royal Society A 367:4779-4799.

Metz, J. A. J., S. A. H. Geritz, G. Meszéna, F. J. A. Jacobs, and J. S. van Heerwarden. 1996. Adaptive dynamics: a geometrical study of the consequences of nearly faithful replication. Pages 183-231 in S. J. van Strien and S. M. Verduyn Lunel, editors. Stochastic and spatial structures of dynamical systems. North Holland, Amsterdam, The Netherlands.

Metz, J. A. J., S. D. Mylius, and O. Diekmann. 2008. When does evolution optimize. Evolutionary Ecology Research 10:629-654.

Metz, J. A. J., R. M. Nisbet, and S. A. H. Geritz. 1992. How should we define fitness for general ecological scenarios? Trends in Ecology and Evolution 7:198-202.

Montarry, J., P. Cartolaro, F. Delmotte, J. Jolivet, and L. Willocquet. 2008. Genetic structure and aggressiveness of 
Erysiphe necator populations during grapevine powdery mildew epidemics. Applied and Environmental Microbiology 74:6327-6332.

Mougou Hamdane, A., X. Giresse, C. Dutech, and M.-L. Desprez-Loustau. 2010. Spatial distribution of lineages of oak powdery mildew fungi in France, using quick molecular detection methods. Annals of Forest Science 67:212.

Mylius, S. D., and O. Diekmann. 1995. On evolutionarily stable life histories, optimization and the need to be specific about density dependence. Oikos 74:218-224.

Schreiber, S., and J. Tobiason. 2003. The evolution of resource use. Journal of Mathematical Biology 47:56-78.

Shaw, M. W. 1994. Seasonally induced chaotic dynamics and their implications in models of plant-disease. Plant Pathology 43:790-801.
Smith, R. J. 2008. Modelling disease ecology with mathematics. American Institute of Mathematical Sciences, Springfield, Missouri, USA.

Tachikawa, M. 2008. Fluctuation induces evolutionary branching in a mathematical model of ecosystems. PLoS ONE 3:e3925.

van den Berg, F., N. Bacaer, J. A. J. Metz, C. Lannou, and F. van den Bosch. 2011. Periodic host absence can select for both higher or lower parasite transmission rates. Evolutionary Ecology 25:121-137.

van den Berg, F., C. A. Gilligan, D. J. Bailey, and F. van den Bosch. 2010. Periodicity in host availability does not account for evolutionary branching as observed in many plant pathogens: an application to Gaeumannomyces graminis var. tritici. Phytopathology 100:1169-1175. 